\title{
COMMUNICATION
}

\section{Etude in vitro des effets de quelques hémostatiques locaux utilisés en chirurgie orale sur l'hémostase par la technique de la thrombinographie.}

\author{
Laurence $\mathrm{S}^{1,2}$, Voirnesson $\mathbf{G}^{1}$, Poitevin $\mathbf{G}^{2}$, Lefèvre $\mathbf{B}^{1}$, Nguyen $\mathrm{Ph}^{2}$ \\ 1 Unité de chirurgie orale - Pôle d'odontologie, CHU de REIMS, 45 rue Cognacq Jay, 51100, REIMS, FRANCE \\ 2 EA 3801, Laboratoire d'hématologie, Hôpital Robert Debré, Avenue du Général Koenig, 51092, REIMS Cedex, FRANCE
}

\section{Introduction}

L'utilisation des hémostatiques locaux s'est largement répandue en chirurgie orale en raison du nombre croissant de patients nécessitant des thérapeutiques antithrombotiques. Bien que l'effet de ces matériaux sur l'arrêt de l'hémorragie soit objectivé cliniquement et largement décrit lors d'expérimentations in vivo, les études analysant leurs mécanismes d'action sur la coagulation sont pratiquement inexistantes (HAS, rapport d'évaluation «hémostatiques locaux» 2011). Les objectifs de ce travail ont été d'évaluer in vitro la capacité pour des matériaux utilisés en chirurgie orale de générer de la thrombine dans des échantillons plasmatiques. Cette génération a été évaluée par une technique de thrombinographie.

\section{Matériel et Méthodes}

La thrombinographie est une exploration fonctionnelle de l'hémostase permettant la caractérisation de la cinétique et de la quantité de travail thrombinique. Elle repose sur l'enregistrement de l'activité de la thrombine in vitro, par fluorométrie (HC Hemker et al 2003). L'intensité du signal recueilli est corrélée à la génération de thrombine par mesure comparative de la fluorescence d'un échantillon standard d'activité thrombinique connue. L'analyse est réalisée par un logiciel.

Les Plasmas Pauvres en Plaquettes (PPP) et les Plasmas Riches en Plaquettes (PRP) ont été obtenus après des centrifugations successives de prélèvements sanguins effectués sur volontaires. Ces plasmas ont été congelés à $-80^{\circ} \mathrm{C}$ et utilisés à $37^{\circ} \mathrm{C}$ dans les 2 heures suivant leur décongélation.

Les matériaux testés ont été le Curasorb ${ }^{\circledR}$, le Surgicel ${ }^{\circledR}$ et le Pangen ${ }^{\circledR}$. Chaque échantillon a été calibré avec un emporte-pièce stérile et déposé au fond des puits de la plaque avant la mise en place des réactifs.
Les résultats obtenus en présence des matériaux sont comparés à ceux de témoins positifs (Plasmas+initiateurs) et de témoins négatifs (Plasmas seuls).

\section{Résultats}

Les différents matériaux testés en présence de PPP et en absence d'initiateurs n'induisent aucune réaction. En ajoutant du Facteur Tissulaire (FT) et/ou des Phospholipides (PPLs), quelques différences apparaissent, le Curasorb ${ }^{\circledR}$ permet une diminution du temps de latence, le Pangen ${ }^{\circledR}$ semble le moins perturber la réaction en présence de FT. Pour le Surgice ${ }^{\circledR}$, les résultats sont inexistants.

En présence de PRP, les matériaux testés seuls permettent l'obtention d'une courbe de génération au profil approchant celui du témoin positif (PRP+FT), équivalent aux résultats obtenus en testant les PRP seuls. Le Curasorb ${ }^{\circledR}$ diminue les temps de latence. Le Surgicel ${ }^{\circledR}$ donne des résultats évoquant la possibilité d'une action au niveau des plaquettes. Le Pangen ${ }^{\circledR}$ génère des courbes au profil identique à celui des témoins avec un temps de latence augmenté.

\section{Discussion}

Cette étude a mis en évidence l'absence d'inhibition des matériaux sur la génération de thrombine. Aucun produit ne permet de promouvoir la coagulation et seuls les effets mécaniques permettent d'expliquer leurs actions sur l'arrêt du saignement. Une action sur les plaquettes semble être mise en évidence en présence de certains des matériaux.

LAURENCE Sébastien sebastien-laurence@univ-reims.fr

This is an Open Access article distributed under the terms of the Creative Commons Attribution License 4.0, which permits unrestricted use, distribution, and reproduction in any medium, provided the original work is properly cited. 